\title{
EDITORIAL
}

\section{Keeping sight of the goal}

\author{
The call by the World Health Organization for all clinical trials to be registered, and for \\ information to be fully disclosed at the time of registration, has antagonized the \\ pharmaceutical industry. More pragmatism could be needed if the important initiative \\ to improve the transparency of clinical research is to maintain momentum.
}

In 2004, the International Committee of Medical Journal Editors (ICMJE), which represents 11 of the world's leading medical journals, announced that its member journals would only consider papers reporting trial results for publication if the trial had been registered at its outset in an acceptable registry. This policy, which was fully implemented last year, has clearly been successful in encouraging the registration of clinical trials. For example, the number of trials registered at the largest acceptable registry - ClinicalTrials.gov at the US National Institutes of Health - now totals nearly 30,000, and has more than doubled since the ICMJE announcement.

Inspired by the same goal as the ICMJE - increasing transparency with respect to the performance and reporting of clinical trials - the World Health Organization (WHO) has recently called for full registration of clinical trials. The WHO also announced that it will launch a registry platform with the aim of bringing registers together in a global network to provide a single point of access to the information stored in them. However, in one key respect, the WHO standpoint is considerably more demanding than that of the ICMJE: it advocates the registration of all clinical trials, not just those that are intended to study the relationship between a medical intervention and a health outcome. In other words, even the registration of Phase I trials in healthy patients, such as those studying drug pharmacokinetics, is considered to be a requirement.

Unsurprisingly, this standpoint has angered the pharmaceutical industry (see the news story on p527 of this issue). Full registration of a clinical trial as defined by the WHO involves the submission of twenty pieces of data, some of which are commercially sensitive, such as the scientific title of the study and the expected outcomes. As well as potentially jeopardizing competitive advantages, industry representatives have highlighted that the requirement of full disclosure of such details at the time of registration, even for early-stage exploratory trials, risks further compromising intellectual property. Both issues, it is believed, could act as a major disincentive for companies to invest in such studies, stifling innovation.
So, could the potential benefits compensate for these potential risks? In the case of later-stage trials, intended to provide evidence of the efficacy and safety of a medical intervention, the benefits of registration are clear. In particular, by ensuring that at least the existence of all such evidence is publicly available, decisions that affect medical practice can take account of all the relevant data about a particular intervention, which typically comes from multiple clinical trials. Indeed, it was the potential of registration to address publication bias, as highlighted in the case of the non-publication of negative or inconclusive trials of antidepressants in paediatric patients, that provided impetus to the ICMJE's initiative.

But in the case of early-stage exploratory trials, the argument for full registration before initiation is far less compelling. For a start, many such trials involve healthy patients, and so are not ever going to be used at a later date to support the efficacy of a particular intervention. And in general, the data generated from exploratory trials are merely intended to guide the design of larger, more rigorous, studies and so much of it has little value for informing medical practice.

One of the most pressing current challenges related to clinical trial registration is simply the development of a system by which relevant information can be readily obtained (see Nature Rev. Drug Discov. 5, 175-176; 2006), not least because such information is currently scattered in more than 300 registries. The registry platform to be launched by the WHO could help greatly in this respect. However, it is questionable whether compounding this challenge by demanding the inclusion of large amounts of exploratory data of limited value is pragmatic at this time. In response to the ICMJE policy, industry has made much progress in improving the transparency of clinical research. At present, maintaining the focus on trials assessing the effects of therapeutic interventions on health outcomes as defined by the ICMJE seems to be a better balance of the needs of all stakeholders in clinical research. And, as such, it is more likely to receive the continued support needed from all parties for the registration initiative to achieve its key goals as soon as possible. 